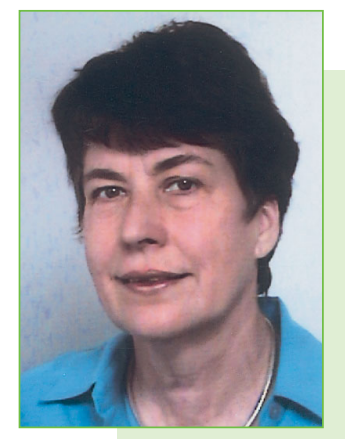

\title{
Sind pflanzliche Arzneimittel anders als Synthetika?
}

Die Frage ist zu bejahen, wenn man von der komplexen Zusammensetzung pflanzlicher Arzneimittel ausgeht. Andere Gründe - wie die Annahme einer im Prinzip anderen Wirkweise - entspringen einer weltanschaulichen Betrachtungsweise und sind irrational. Ein grundsätzlicher Unterschied lässt sich allerdings in der Einstellung zu Phytopharmaka feststellen. Eine sachliche, emotionslose Einstellung ist sehr selten, die meisten sind entweder "dafür» oder "dagegen". Und diese Polarisierung zieht sich durch alle Ebenen vom mehr oder weniger gut informierten Verbraucher bis zur wissenschaftlichen Diskussion, bei der häufig von vorher festliegenden Standpunkten aus diskutiert wird. Das erschwert eine Verständigung, um es vorsichtig zu formulieren, und dient der Sache sicher nicht.

Aber einiges hat sich doch geändert. Vor 25 Jahren gab es erbitterte Diskussionen darüber, ob man einer Pflanze so etwas »antun« könnte wie eine Prüfung in denselben Modellen wie Synthetika. Das ist - meiner Meinung nach zum Glück - vorbei. Eine solche rein weltanschauliche Betrachtungsweise von Phytopharmaka ist nicht nötig, um ihr Dasein zu rechtfertigen. Im Gegenteil, sie macht sie verdächtig.

Aber eine andere Besonderheit von Phytopharmaka wird odt vergessen und das kann sich sehr nachteilig auswirken. Phytopharmaka sind Vielstoffgemische, bei denen häufig mehrere Inhaltsstoffe zur Gesamtwirkung beitragen. Immer wieder wird, sobald ein Inhaltsstoff identifiziert ist, der ähnlich wirkt wie der Gesamtextrakt, dieser zu dem Wirkstoff erklärt. Dabei fehlen in den meisten Fällen Untersuchungen zu dosisabhängigen Effekten von Ausgangsextrakt und dem neuen Inhaltsstoff, die zeigen, dass sich tatsächlich die Wirkung des Extraktes in quantitativer und qualitativer Hinsicht durch diesen einen Inhaltsstoff erklären lässt. Am
Beispiel Salix lässt sich zeigen, warum die Fokussierung auf einen einzelnen Inhaltsstoff nachteilig sein kann. Salicin-Derivate wurden als die Wirkstoffe von Salix-Zubereitungen angesehen; sie sind aber in zu geringer Konzentration enthalten, um deren analgetische und antiphlogistische Wirkung zu erklären. Deshalb wurde häufig geschlossen, dass solche Präparate nicht ausreichend wirksam sein können. In eigenen Untersuchungen konnten als weitere Wirkqualitäten solcher Extrakte eine sehr ausgeprägte antidepressive und eine anxiolytische Wirkung beobachtet werden. Damit ergibt sich eine rationale Erklärung für die Wirkung solcher Präparate. Antidepressiva werden in der chronischen Schmerztherapie zur effektiven Verstärkung der Wirksamkeit mit Nicht-Opioid- und mit OpiodAnalgetika kombiniert.

Ob damit das Wirkspektrum von Salix-Zubereitungen vollständig erfasst ist, oder ob noch ganz andere Wirkqualitäten das Wirkspektrum ergänzen, lässt sich noch nicht sagen. Aber an diesem Beispiel wird deutlich, dass der Ersatz eines Extraktes durch einen einzelnen Inhaltsstoff häufig ebenso wenig wünschenswert und sinnvoll ist wie die Fokussierung auf einen einzelnen Inhaltsstoff eines Extraktes. Das ist sicher ein Unterschied zu Synthetika, der die Untersuchungen mit Phytopharmaka schwierig und faszinierend macht.

1930 zitiert G. Volk Huchard (Hippokrates 5/6, 293): "Die Medizin soll eine Schule der Duldsamkeit und insbesondere der Bescheidenheit sein, aus Gründen, die uns, ach, nur zu gut bekannt sind; sie soll entgegengesetzten Theorien gegenüber keine abwehrende, stolze Haltung einnehmen, weil kein Einzelner und keine Schule glauben darf, allein im Besitze der Wahrheit zu sein."

Hilke Winterhoff 